\title{
Fäkalogie oder Ausscheidung, Microbiom und ich
}

Peter Marko

Korrespondenz: Dr. med. Peter Marko Bruggwaldstrasse 39e CH-9009 St. Gallen

p.marko[at]bluewin.ch
Wie jedes neugierige Kleinkind beobachtete und bewunderte auch ich früher meine Ausscheidung. Ich ging nicht so weit wie eine Cousine, die unverbesserlich und uneinsichtig mehrmals mit ihr spielte und sie herumschmierte. Vermutlich wurde mir beim ersten solchen Versuch unmissverständlich beigebracht, dass sich so etwas, wie vieles andere, nicht gehört und mit Strafe gedroht wurde - wie damals bei einer guten Erziehung üblich, der ich unbestreitbar ausgesetzt war.

Später, nicht mehr auf dem Topf, bekam ich sie nicht mehr zu Gesicht, da ich mein Bedürfnis auf dem Plumpsklo im Hof verrichten musste. Dort verschwand sie und verlor ihre Eigenschaften in der einheitlichen Brühe, in der sich verschiedene kleine Lebewesen unermüdlich tummelten. Wir hatten zwar auch ein WC im Badezimmer, aber das durften nur Gäste benützen und wir, wenn wir krank waren, oder wenn draussen Kälte unter $-10^{\circ} \mathrm{C}$ herrschte. Dann schliefen ausnahmsweise auch unsere Katzen im Windfang.

Diese harte Massnahme war gutbegründet. Man sollte den störanfälligen Abfluss nicht verstopfen mit den kleingeschnittenen Zeitungen, die man zur Reinigung brauchte, da das Toilettenpapier während des Krieges schwierig zu besorgen und kostbar war, und die eben nur für die Gäste kurz vor ihrer Ankunft ins WC gelegt wurden. Das Badezimmer, dazu ohne Fenster, befand sich zwischen zwei Zimmern, und vor allem ein Kind sollte man früh genug für das Leben abhärten und nicht unnötig verwöhnen. Damit lernten wir auf natürliche Weise unsere Ausscheidung zu beherrschen und zu steuern, da wir

\section{Ich verkündete, ein Fäkalist werden zu wollen.}

unser Geschäft aus Angst vor Beginn der Dunkelheit und speziell in den kalten Jahreszeiten schnell erledigen wollten. Meine kurzen Beine hingen in der Luft, und die Umstände waren auch sonst einem längeren Aufenthalt nicht zugeneigt, bei dem man ruhig in illustrierten Kinderbüchern hätte blättern, später lesen mögen. In den warmen Jahreszeiten störten dabei, eher aus Übermut, Gewohnheit und Bewegungsdrang, die sicher nicht mehr hungrigen Fliegen.
Erst nachdem wir in ein vierstöckiges modernes Haus in der Hauptstadt umgezogen waren, konnte ich meine Beobachtungen der Ausscheidung fortsetzen. Ich merkte, dass ihre Häufigkeit, Form, Farbe, Beschaffenheit, ihr Geruch wie auch die Empfindungen vor, während und nach ihr wechselten und von verschiedenen Umständen abhingen und beeinflusst wurden. Ausser Nahrung und Bewegung, die als solche schon anerkannt waren, schienen mir unter anderem auch seelische Einflüsse eine Rolle zu spielen. Ich bemerkte, falls alle Voraussetzungen erfüllt waren, drängt die Ausscheidung nicht überstürzt, mit unangenehmem Druck oder sogar Schmerzen nach aussen, sondern klopft leise, höflich an und wartet, bis alle Umstände für ihr Erscheinen günstig sind. Dann schlüpft sie glatt, gutgeformt, ohne unangenehmen Geruch raus und hinterlässt eine Erleichterung, nicht nur im Bauch, sondern von Kopf bis Fuss. Es wurde mir auch bewusst, dass die Ausscheidung nur für andere Menschen unangenehm, mitunter ekelhaft stinkt. Es ist ein Zeichen einer guten, gesunden, zweckmässigen Eigenliebe. Sonst würden wir ihren Verlauf zu früh abbrechen, was, wenn wir es manchmal nicht vermeiden können, nicht nur unangenehm, aber wenn es sich häuft, auch schädlich sein kann. Der Ekel vor einer fremden Ausscheidung scheint aber ein späteres Zeichen der Zivilisation zu sein, die in diesem Falle unsere Sitten vernünftig verfeinerte. Im Mittelalter warf man noch hemmungslos und rücksichtslos den Stuhl auf die Strasse und spülte ihn in die Gewässer, aus denen man, ahnungslos über die schädlichen, bösen Folgen solches Tuns, Trinkwasser entnahm. Vielleicht konnte man ihn auf solche technisch einfache Weise entsorgen, weil wir uns an den jeweiligen Gestank schnell gewöhnen. Unser Geruchssinn stumpft ab. Ein gewisser Ekel war aber immerhin vorhanden: Warum benützten wohl die alten Ärzte dieses Ausscheidungsprodukt nicht wie den Urin zu diagnostischen Zwecken? Warum trieben sie nicht ähnlich der «Urinschau» auch eine «Stuhlschau»? Die Sprachwissenschaftler und Anthropologen könnten zusammen die Antwort liefern, wenn sie herausfinden würden, seit wann man in welcher Kultur Scheisse als Schimpfwort verwandte. Wie dem auch sei, ich wollte das Versäumnis nachholen und die Stuhlschau mit neuen wissenschaftlichen Methoden gründlich betreiben.

Nachdem ich Medizin zu studieren begann, wurde mir noch bewusster, wie einseitig und beschränkt es ist, wenn man den Stuhl nur mikrobio- 


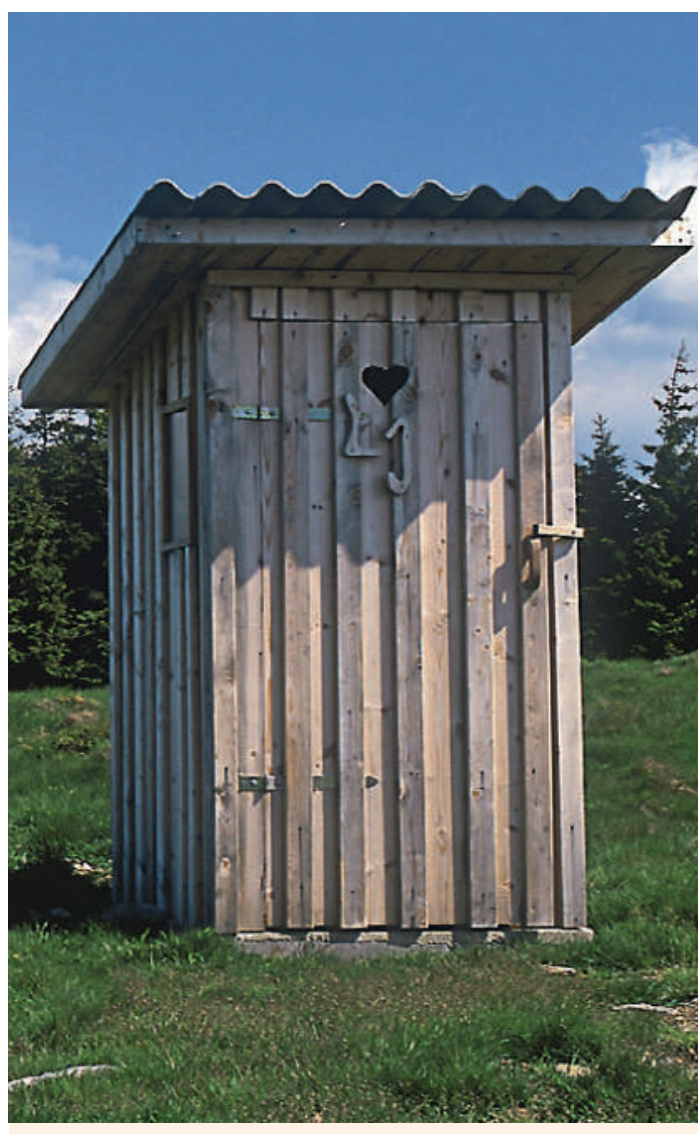

Das neue WC im Haus war nur für die Gäste ...

logisch untersucht. Man kann doch viel mehr daraus ablesen, erfahren als nur, welche Bakterien uns krankmachen. Ich äusserte diese Gedanken meinen Freunden gegenüber und verkündete, ich beabsichtige, ein Fäkalist zu werden. Ich hoffte, ich bin nicht der Einzige auf der Welt mit diesen Interessen, aber in der Vorinternetära konnte ich die Geistesverwandten nicht eruieren. Ich wurde weder ein Gastroenterologe noch ein Proktologe. Sie widmen sich mehr den Organen der Ausscheidung und den Umständen, den Bedingungen, unter denen der Darminhalt produziert wird, und kümmern sich, ausser bei gewissen Infektionskrankheiten und Verdauungsstörungen, kaum darum, was er uns vermittelt, was er aussagt. Ich dachte, man bräuchte eine neue Richtung, eigentlich eine Metawissenschaft, die sich dem Inhalt des Darmes, diesem noch nicht geborgenen Schatz an Informationen, ganzheitlich, holistisch widmen würde - eben eine Fäkalogie.

Die Wende zum Besseren kam vor rund zehn Jahren. Sie hängt, wie nicht selten in der Wissenschaft, mit der Entwicklung neuer Methoden zusammen. Mit Hilfe der Gentechnik kann man relativ einfach die Zusammensetzung der Mikrobenarten im Darminhalt bestimmen. Damit entdeckte man, dass Mikroorganismen nicht nur Infektionen verursa- chen, sondern sich auch an der Entstehung anderer Krankheiten beteiligen und im günstigen Fall unsere Gesundheit unterstützen. Schon unsere Geburt, später der Lebensstil, vor allem die Nahrung beeinflusst die Zusammensetzung der Mikroorganismen im Darm. Je vielfältiger die Bakterien in Darm sind, desto besser. Das Microbiom des Darmes, wie man die Ansammlung der verschiedenen Mikroorganismen nennt - ähnliche gibt es auch auf der Haut und den Schleimhäuten - wirkt sich aus auf das Gewicht, das Aussehen, unseren seelischen und körperlichen Zustand und ändert auch die Wirkung der Medikamente. Man benützt diese Kenntnisse bereits für die Therapie vieler Krankheiten, die sonst schwierig zu behandeln sind. Dabei transplantiert man den gereinigten Stuhl der Gesunden durch eine Sonde oder in Kapseln in die Kranken, z. B. bei verschiedenen Autoimmunkrankheiten. Es gibt Therapeuten, die bereits mehr als tausendmal den Stuhl transplantierten, angeblich mit durchschlagendem Erfolg und zur grossen Zufriedenheit und Dankbarkeit der Patienten. Manche wollen nur die günstigen Mikroorganismen isolieren, züchten und dann verabreichen. Andere meinen, dass eben die Mischung, die Vielfalt der Mikroorganismen, heilsam wirkt. Im Darminhalt gibt es noch um mehrere Zehnerpotenzen mehr Viren als Bakterien. Man beginnt auch darüber zu forschen, was sie mit uns tun, wie sich ihre Menge und Zusammensetzung auf uns auswirkt. Wie nicht selten am Anfang der Entwicklung einer Disziplin mischt sich noch die «Schulmedizin» mit ihren strengen Regeln und Kriterien mit der freieren, wilden, weniger regulierten Erfahrungsmedizin, die sich einfach auf Erfolg der Therapie richtet. Wir wissen aber noch nicht, wie sie sich langfristig auswirkt. Verursacht sie wirklich keine unerwünschten Wirkungen, weil die Bakterien bei den ursprünglichen Wirten, den «Stuhldonoren», natürlich vorkommen?

Abhandlungen über Atome beginnen gewöhnlich mit Demokrit. Hätte ich meine Beobachtungen und Gedanken schon früher veröffentlicht - aber wer, welche Zeitschrift wäre damals dazu bereit gewesen - würde ich vielleicht in Arbeiten über Microbiom des Darmes auch erwähnt. Das Leben ist, wie ein weiser Mensch sagte, eine Reihe von Versäumnissen, Versagen und Niederlagen. Wir lernen, damit umzugehen und es zu ertragen.

In unserem WC mit Closomat haben wir eine reichliche Auswahl an Zeitschriften und Büchern und auch Zündhölzchen gegen eventuellen schlechten Geruch, weil dieser durch das Feuer vernichtet wird. Man kann dort entspannt, ruhig verweilen und dem Stuhlgang seinen natürlichen Lauf lassen, beobachten und nachdenken, so wie man es in der frühen Kindheit ungestört auf dem Topf gerne tat. Habe ich es nicht im Leben ziemlich weit gebracht? Oder haben sich nur die Zeiten geändert? 